\title{
Propuesta preliminar de incorporación de nuevos sitios de investigaciones socio-ecológicas de largo plazo en la Red LTSER de Chile
}

\author{
Preliminary proposal to incorporate new sites for long-term socio-ecological research \\ into the Chilean LTSER network
}

\author{
Pablo J Donoso ${ }^{a *}$, Jennifer C Zavaleta ${ }^{a}$ \\ *Autor de correspondencia: ${ }^{a}$ Universidad Austral de Chile, Facultad de Ciencias Forestales y Recursos Naturales, Instituto de \\ Bosques y Sociedad, Isla Teja s/n, Valdivia, Chile, tel.: 56-632221056, pdonoso@uach.cl
}

\begin{abstract}
SUMMARY
Currently Chile belongs to the International Long Term Ecological Research network (ILTER) through three sites: Bosque Fray Jorge National Park, Senda Darwin Biological Station, and Omora Ethnobotanic Park. At the end of 2012 a workshop was held in the Las Animas Cascade Nature Sanctuary with the aim of exploring the potential inclusion of other long-term ecological research sites into the so-called Long-Term Social-Ecological Research sites of Chile (LTSER-Chile). One of the conclusions of this workshop was that it is necessary to know with certain depth what is each site doing in terms of research, education, and outreach. As a consequence, at the beginning of 2013 we conducted a survey to 13 sites, including the three foundational sites and 10 potential sites identified during the workshop. Once received the answers from 12 of the 13 sites, we found that these sites are concentrated mainly in the Valdivian Ecoregion (39-42 $\left.{ }^{\circ} \mathrm{S}\right)$, and the research activities are related mainly to terrestrial ecology and marine ecology in two cases. Almost all sites had activities during the last decade, few sites had long-term research, most sites have conducted outreach activities, but no site had socioecological research. The results show the potential of the sites to extend the LTSER-Chile, but illustrate the need to establish future criteria to belong to the network and the need to include sites in Ecoregions not represented so far.
\end{abstract}

Key words: ecoregions, Valdivian forests, outreach.

\section{RESUMEN}

En la actualidad Chile pertenece a la red internacional de sitios de estudios ecológicos de largo plazo (ILTER del inglés International Long Term Ecological Research Network) a través de tres sitios: Parque Nacional Bosque Fray Jorge, Estación Ecológica Senda Darwin y Parque Etnobotánico Omora. A finales del año 2012 se efectuó un taller en el Santuario de la Naturaleza Cascada de Las Ánimas con el objetivo de explorar potenciales nuevos sitios que pudiesen incorporarse a LTSER-Chile. Una de las conclusiones de ese taller fue que es necesario conocer con cierto nivel de profundidad lo que cada sitio ha estado haciendo en términos de investigación, educación y vinculación con el medio. En consecuencia, a principios de 2013 se efectuó una encuesta que fue dirigida a 13 sitios, incluyendo los tres actuales de la red más otros 10 identificados en el taller. Recibidas las respuestas de 12 de los 13 sitios, la información sistematizada permite visualizar que estos se concentran fundamentalmente en la ecorregión de los bosques Valdivianos $\left(39-42^{\circ} \mathrm{S}\right)$, concentran sus investigaciones en ecología (terrestre en general y en dos casos marina) pero fundamentalmente en la última década (hay pocos sitios con investigaciones de largo plazo) y carecen de estudios socioecológicos a pesar de que la mayoría manifiesta tener actividades de vinculación con el medio. Los resultados muestran el potencial de sitios para ampliar la LTSER-Chile, pero ilustran la necesidad de establecer a futuro criterios para pertenecer a la red y la necesidad de incluir sitios de ecorregiones no representadas aún.

Palabras clave: ecorregiones, bosques valdivianos, vinculación con el medio.

\section{INTRODUCCIÓN}

La propuesta de una Red de Investigación Socio-Ecológica Largo Plazo (LTSER por sus siglas en inglés) corresponde a un iniciativa interdisciplinaria que se centra en las ciencias básicas y naturales y que se extiende por escalas temporales, y más recientemente también por el tema de las dimensiones humanas de los ecosistemas (Haberl et al. 2006). Desde los inicios del primer programa nacional de investigación ecológica de largo plazo en los Estados Unidos en 1980, la ILTER ha expandido no sólo su ámbito conceptual sino también su rango geográfico para incluir más sitios y países en distintos ecosistemas para comparar y avanzar en el conocimiento de las funciones del clima y los ecosistemas en el largo plazo. Algunas regiones en el hemisferio sur, como aquellas en Chile, han estado poco representadas, a pesar de su importancia en la ILTER ya que la posición geográfica de estos sitios corresponde a 
lugares en el planeta que no han estado expuestos a niveles significativos de contaminación atmosférica, $y$, por lo tanto, no tienen depósitos de nitrógeno atmosférico y otros contaminantes industriales. Por lo tanto, las regiones del sur de Sudamérica constituyen una línea base para el entendimiento de los ciclos biogeoquímicos antes de la revolución industrial (Anderson et al. 2012, Hedin et al. 1995). Además, en Chile, se encuentran ecosistemas de gran diversidad y relevancia como los bosques templados lluviosos y los humedales templados más grandes del hemisferio sur, así como también los más grandes campos de hielo, fuera de la Antártida y Groenlandia (Anderson et al. 2012).

Aunque en Chile ya existen investigaciones de largo plazo en varios sitios, Chile se convirtió parte de la ILTER en el año 2010 únicamente con tres sitios: el Sitio Experimental Fray Jorge en la región mediterránea, la Estación Biológica Senda Darwin en la región de la Selva Valdiviana o de bosques templados lluviosos Norpatagónicos, y el Parque Etnobotánico Omora en la región subantártica o de Bosques Templados Magallánicos (Anderson et al. 2010) que eran coordinados por la Corporación Instituto de Ecología y Biodiversidad (IEB). Por lo tanto, su reconocimiento como red nacional ha tenido el compromiso de generar mecanismos para ampliar y legitimar la red tanto en su conformación de instituciones y grupos de trabajo como en la representatividad geográfica del país. Es así que en la actualidad, la Red LTSER-Chile ha tomado la iniciativa de incrementar el número de sitios para representar de manera más integral todas las ecorregiones del país y también involucrar a una mayor diversidad de actores. El presente trabajo ofrece un primer análisis de la situación actual de los tres sitios actuales más nueve nuevos sitios potenciales para ser incluidos en Red LTSER-Chile en términos de investigación, infraestructura, educación y divulgación, y vinculación con el medio. Los resultados de este análisis permiten evaluar la representatividad ecológica de estos sitios, su actual o potencial impacto en investigación ecológica y social, así como los desafíos de LTSER-Chile en términos de llegar a constituir una red representativa e inclusiva de los ecosistemas naturales e instituciones actualmente abocados a la investigación de largo plazo en Chile.

\section{METODOLOGÍA}

En base a una lista de invitados al taller Fortalecimiento de la Red Chilena de Sitios de Estudios Socio-Ecológicos de Largo Plazo, se envió una encuesta estructurada (https:// www.surveymonkey.com/s/8GWTVQ5) a doce personas que representan los tres sitios actuales de la red LTSERChile y otros nueve sitios potenciales (figura 1) a principios de 2013. La mayoría de los sitios, salvo el Parque Nacional Bosque Fray Jorge (PNBFJ) y el Parque Etnobotánico Omora, son privados (cuadro 1). Nueve sitios pertenecen a universidades, cuatro a la Pontificia Univer-

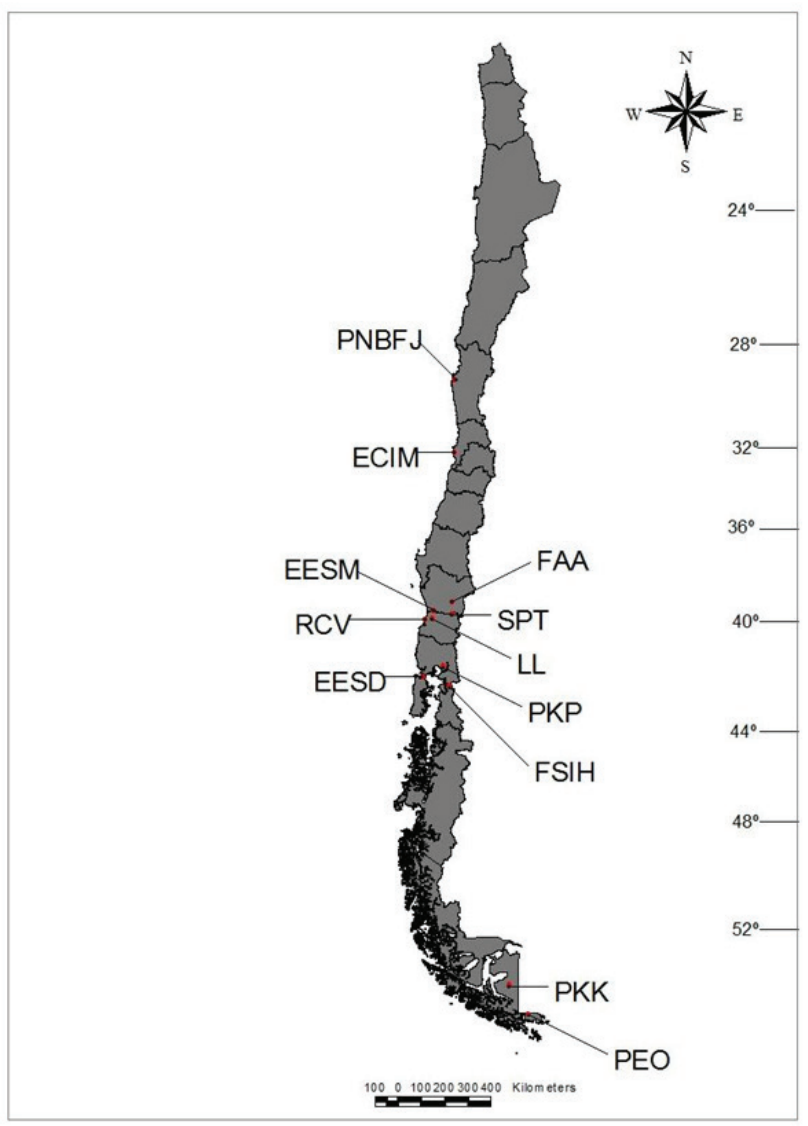

Figura 1. Ubicación geográfica de los sitios actuales y potenciales de la LTSER-Chile. El nombre completo de cada sitio se encuentra en el cuadro 1.

Geographic location of existing and potential LTSER-Chile sites. The full name of each site is given in table 1.

sidad Católica de Chile, tres a la Universidad Austral de Chile, uno a la Universidad Católica de Valparaíso y uno a la Universidad de Magallanes. Adicionalmente, dos sitios pertenecen a ONG internacionales y un sitio es particular.

La encuesta tuvo 32 preguntas sobre temas de información general, investigaciones, infraestructura, educación y difusión, y vinculación con la sociedad. Estos temas fueron identificados durante el taller, incluyendo los aspectos más importantes para que un sitio cumpla con los requisitos para estudios socio-ecológicos de largo plazo. Después de un estudio piloto con dos sitios, se hicieron ajustes para clarificar preguntas establecidas originalmente y se les pidió a los encuestados que respondieran dentro de un mes desde el momento en que les fueron enviadas las encuestas (abril de 2013). Hubo sólo una institución que no respondió. La encuesta preguntó en primer lugar cuáles eran los objetivos de la investigación en los sitios, siendo las categorías generales conservación, preservación, restauración, manejo, turismo, producción, y educación, y en segundo lugar en forma más específica se preguntó por disciplinas de investigación de largo plazo (> 10 años) y de corto 
Cuadro 1. Listado de actuales y potenciales sitios socioecológicos de largo plazo para ser considerados en la LTSER-Chile, ordenados de norte a sur.

List of current and potential long-term socioecological sites to be considered in the LTSER-Chile, ordered from north to south.

\begin{tabular}{lll}
\hline \multicolumn{1}{c}{ Sitio } & \multicolumn{1}{c}{ Propiedad o administración } & Sigla \\
\hline Parque Nacional Bosque Fray Jorge & CONAF, Gobierno de Chile & PNBFJ \\
Estación Costera de Investigaciones Marinas & Pontificia Universidad Católica de Chile & ECIM \\
Fauna Australis Araucanía & Pontificia Universidad Católica de Chile & FAA \\
Estación Experimental Bosque San Martín & Universidad Austral de Chile & EESM \\
San Pablo de Tregua & Universidad Austral de Chile & SPT \\
Llancahue & Universidad Austral de Chile & LL \\
Reserva Costera Valdiviana & The Nature Conservancy & RCV \\
Parque Katalapi & Particular & PKP \\
Fundación San Ignacio de Huinay & Universidad Católica de Valparaiso & FSIH \\
Estación Ecológica Senda Darwin & Fundación Senda Darwin y Pontificia Universidad Católica de Chile & EESD \\
Parque Etnobotánico Omora & Fundación Omora y Universidad de Magallanes & PEO \\
Parque Karukinka & Wildlife Conservation Society & PKK \\
\hline
\end{tabular}

plazo ( $<10$ años). Además se consultó por infraestructura para investigación, con énfasis en instrumentos para medición de clima ya que esta es una variable fundamental de registrar en estudios de largo plazo, y, finalmente, por productos de investigación expresados en publicaciones y tesis de pre y postgrado. En un segundo bloque se encuestaron aspectos relacionados con educación a través de la realización de cursos y talleres, de la generación de folletería, y de la existencia de infraestructura para actividades de educación ambiental, así como actividades de vinculación con el medio, expresadas a través de diferentes vínculos con la sociedad y las comunidades vecinas, incluyendo actividades productivas. Finalmente, se consultó acerca de la infraestructura de los sitios en términos de acceso, alojamiento e instalaciones de oficinas para trabajar. La información entregada corresponde exclusivamente a aquella entregada por quienes respondieron las encuestas en representación de los sitios respectivos.

\section{RESULTADOS}

Muchos de los sitios se encuentran en las regiones de Los Ríos (XIV) $(n=4)$ y de Los Lagos (X) (n=3), es decir en la ecorregión de bosques templados lluviosos Valdivianos, aunque en la parte sur de la región de Los Lagos también se les reconoce como bosques templados lluviosos Norpatagónicos (Gutiérrez y Huth 2012; cuadro 2). Todos los sitios tienen objetivos de conservación y educación, con la excepción de Fundación San Ignacio del Huinay que no tiene el objetivo de educación. La mayoría de los sitios participan en turismo $(n=9)$ y restauración $(n=8)$. Los sitios con investigaciones de largo plazo $(n=10)$ se focali- zan en varios temas, pero ecología vegetal $(n=6)$ y animal $(n=4)$ son los temas más comunes. Los tres sitios actuales de la LTSER-Chile tienen estaciones climatológicas, investigaciones de clima, y junto con el ECIM son los únicos sitios con datos climatológicos de largo plazo. En términos de investigación a corto plazo, en todos los sitios existen investigaciones en ejecución. Los investigaciones más comunes fueron en ecología vegetal ( $n=9)$, ecología forestal $(n=9)$, ecología animal $(n=7)$, cambio climático $(n=6)$, clima $(n=4)$, y ecología de paisaje $(n=4)$. En los últimos tres años, todos los sitios tenían publicaciones con arbitraje y todos tenían tesis de pre-grado y post-grado, con la excepción de FSIH. Cada uno de los tres sitios actualmente en LTSER-Chile, además de otros tres sitios de la lista, tenían al menos diez publicaciones con arbitraje (cuadro 2).

El acceso a los sitios no es un problema en ninguno de los sitios, aunque durante los períodos más lluviosos en el año algunos sitios son poco accesibles. Todos los sitios tienen sala de trabajo $(n=9)$ o para talleres $(n=9)$ a excepción de LL. Además, todos los sitios, a excepción de Huinay, han organizado cursos o talleres, con siete sitios que han organizado más de tres cursos o talleres por año. Dos de los sitios actuales de la LTSER-Chile más siete sitios expresaron tener vínculos con la sociedad. En sus respuestas, los representantes de los sitios indicaron que sus vinculaciones se referían a trabajos con colegios, turismo, generación de productos como leña para la comunidad, y provisión de visitas de médicos, así como acceso gratuito a electricidad. Algunos sitios tenían relaciones formales con la comunidad y se reunían regularmente en forma diaria, mensual, o una vez cada ocho meses. Otros sitios declararon tener interacciones informales e irregulares con la comunidad. 


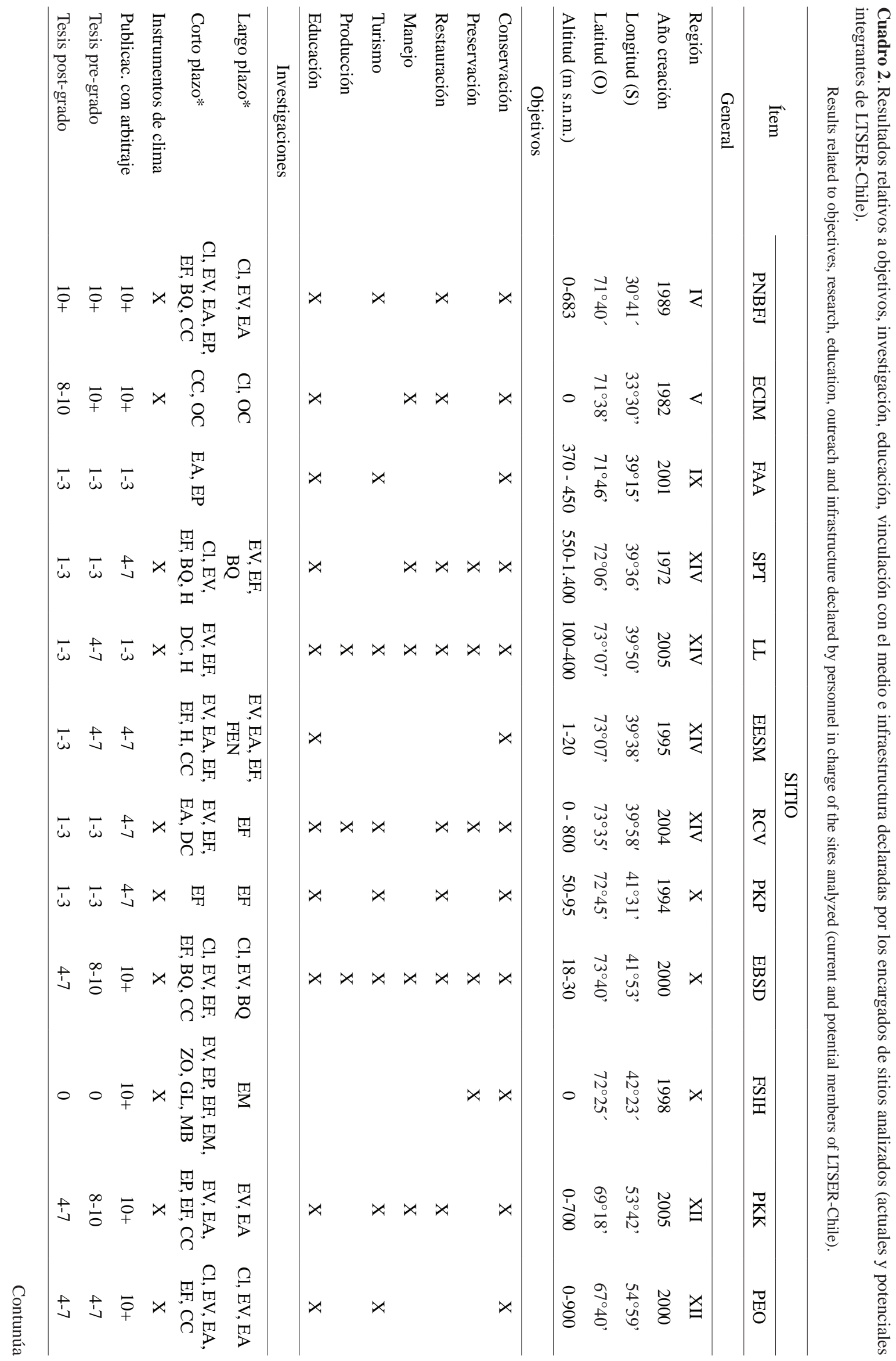




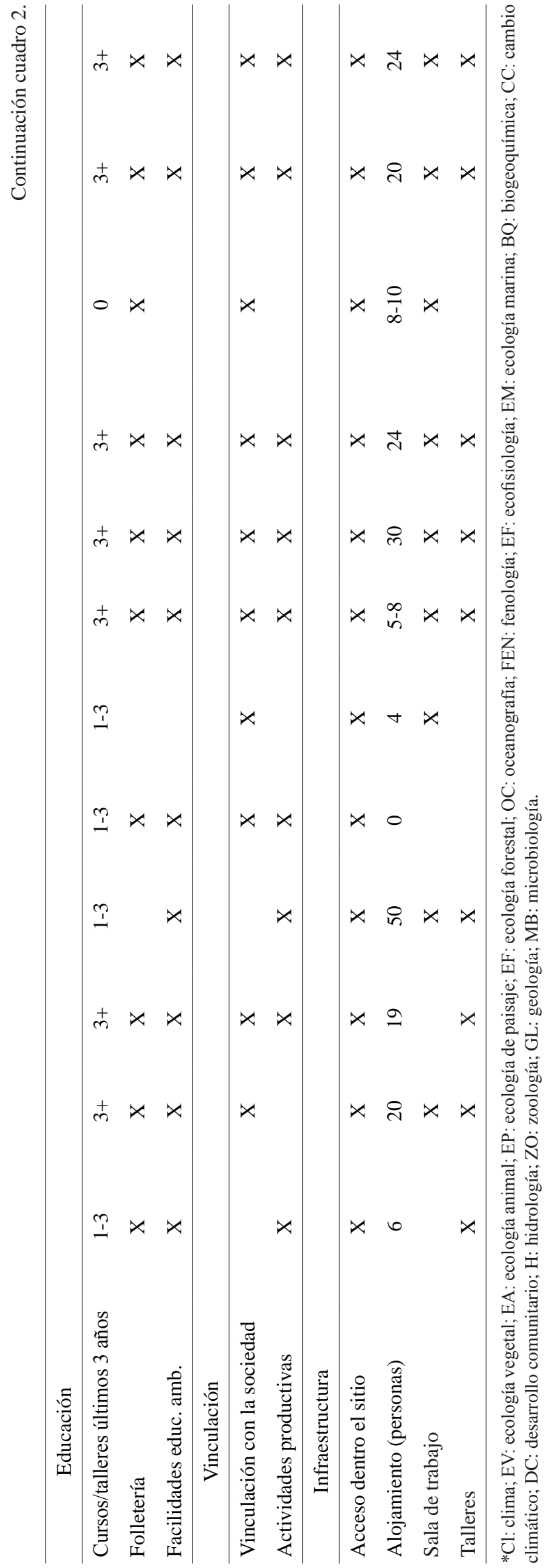




\section{DISCUSIÓN}

La investigación desarrollada en la actualidad en los 12 sitios indicados en el cuadro 2 refleja que hay múltiples líneas de investigación en desarrollo, las cuales se categorizaron en 16 disciplinas. La mayoría de los sitios reporta varias disciplinas de investigación en el corto plazo ( $<10$ años) pero pocas en un plazo más largo ( $>10$ años). Este análisis demuestra que los sitios potenciales para ser incluidos en la Red LTSER-Chile representan un complemento relevante a aquellos tres sitios que actualmente el IEB apoya. En primer lugar, con estos nuevos sitios se expanden las ecorregiones representadas, al incluirse en especial sitios en regiones más al norte dentro de la ecorregión de bosques templados lluviosos Valdivianos. En segundo lugar, entre los sitios señalados en el cuadro 2 se incluyen dos que se dedican a investigaciones de ecología marina, una línea de trabajo que hasta la fecha no ha sido incluida en la LTSER-Chile a pesar de la cercanía de aquellos tres sitios con el océano Pacífico (figura 1). En tercer lugar, se diversifica el tipo de instituciones y propietarios potenciales a ser incluidos en la red. En la actual red LTSER-Chile, de los tres sitios dos pertenecen al Estado de Chile (público), de los cuales uno de ellos es administrado por la Corporación Nacional Forestal (CONAF) y el otro por una Fundación sin fines de lucro (público-privado); el tercer sitio es de propiedad y administrado por una Fundación sin fines de lucro (privado). En el listado del cuadro 2 aparecen otras universidades, un sitio de pequeño tamaño de propiedad particular y dos sitios pertenecientes a grandes ONG mundiales ambientalistas.

Los sitios encuestados en este estudio, son una muestra de una serie de otros sitios que también realizan investigaciones de largo plazo, o que se han establecido recientemente pero con perspectivas de trabajo de largo plazo, especialmente, sitios en las tres categorías de públicos, privados universitarios y privados particulares. Por lo tanto, es importante que en el proceso de definir una Red LTSER-Chile con representación nacional real se identifiquen más sitios potenciales al menos al nivel desarrollado en el cuadro 2 y conocer los objetivos de investigación y desarrollo de estos sitios. Un especial énfasis se deberá poner en invitar a sitios ubicados en ecosistemas ausentes hasta la fecha, como el desierto, los sistemas alto andinos, los bosques Mediterráneos esclerófilos (33-37 $\left.{ }^{\circ} \mathrm{S}\right)$, y los bosques templados lluviosos del norte de la ecorregión Valdiviana (37-39 $\mathrm{S})$, donde hay una transición hacia los bosques Mediterráneos y que corresponde a la región con mayor diversidad, endemismos, y especies amenazadas del país (Armesto et al. 1998, Bannister et al. 2012). En todo caso, considerando la red actual y los nuevos sitios potenciales, queda en evidencia que la ecorregión de bosques valdivianos acapara mucho interés y se puede convertir en la primera plataforma o polo de sitios de investigación socioecológica de largo plazo. Este estudio, en consecuencia, también permitió identificar vacíos de investigación, es de- cir, la mayoría de los sitios se encuentran en el sur de Chile y, salvo EDIEM, no hay sitios de largo plazo en la zona mediterránea de Chile, donde es urgente impulsar la creación de un sitio para estudios socio-ecológicos de largo plazo.

Por otro lado, cabe señalar que el esfuerzo de construcción de una Red LTSER-Chile más completa y representativa debe entenderse como un proceso dinámico, y se debe transparentar y legitimar tanto en la forma de definir criterios para integración de nuevos sitios como en la búsqueda de alternativas para mejorar la investigación de largo plazo. Las instituciones aspirantes a incorporarse a la Red LTSERChile deben cumplir ciertos requisitos, como los incluidos en el cuadro 2 como umbrales mínimos. Desde este punto de vista, pertenecer a la Red LTSER-Chile debe ser un reconocimiento al trabajo previo y también un compromiso a futuro, $y$, por lo tanto, debe ser acompañado de requisitos mínimos en cuanto a investigación, educación, vinculación con el medio y un manejo moderno y riguroso de las bases de datos generadas. Por otra parte, este compromiso con la investigación debe ser acompañado con avances concretos en la integración de las dimensiones sociales de los ecosistemas y la inclusión real de disciplinas como la antropología, la sociología, la arqueología, entre otros.

Finalmente, es una realidad que la investigación de largo plazo nunca ha tenido financiamiento estable en Chile y no ha sido una meta de CONICYT, su principal organismo de apoyo. Este es un tema fundamental a resolver. La iniciativa liderada por el IEB ha sido innovadora en este sentido para usar fondos de algunos grandes proyectos 0 programas altamente competitivos (por ejemplo, Iniciativa Científica Milenio y el Programa de Financiamiento Basal), pero una solución sistemática es necesaria para otros sitios y programas. En general, las investigaciones en Chile se sostienen fundamentalmente a través de proyectos que en promedio tienen tres años de duración, aunque hay creciente interés de instituciones de investigación por implementar investigaciones de largo plazo, siendo el esfuerzo de ampliar la red coordinada por el IEB un ejemplo. Para mantener y desarrollar una Red LTSER sólida y relevante para Chile y el mundo, no es posible continuar con el actual modelo de financiamiento de la investigación si a ella se le quiere dar una perspectiva de largo plazo para el país. El mismo fortalecimiento de un programa nacional de LTSER debería ser un mecanismo para que haya fondos del Estado chileno para el desarrollo de estas investigaciones.

El presente esfuerzo es la primera iniciativa concreta para profundizar el estudio de potenciales candidatos para incorporarse a la Red LTSER -Chile, respondiendo al llamado hecho por Anderson et al. (2012) de comenzar a formalizar programas de LTSER a nivel nacional en Chile y Argentina, como forma de promover la colaboración entre programas y sitios. Se espera también ver avances de esta naturaleza en el país vecino, pero en cuanto a Chile la información entregada en este estudio y expresada en el cuadro 2 , parece relevante como un avance hacia la identificación de condiciones básicas para optar a integrarse a la Red. Sin 
embargo, se considera necesario también en alguna etapa conocer las perspectivas de investigación de los sitios, tanto en aspectos ecológicos como sociales. Los resultados de la encuesta muestran que la mayoría de las investigaciones de los sitios se asocian a líneas de investigación un tanto clásicas, en especial ecología, y que prácticamente no hay investigación en temas sociales y, en particular, en aquellos que vinculan aspectos ecológicos y sociales. Este es un gran desafío, ya que es fundamental avanzar en llenar el histórico vacío que hay entre las investigaciones científicas y la sociedad, es decir, los usuarios de los recursos y los tomadores de decisiones que afectan el uso de los recursos (Knight et al. 2006, Anderson et al. 2008, Sunderland et al. 2009).

\section{REFERENCIAS}

Armesto JJ, R Rozzi, C Smith-Ramirez, MTK Arroyo. 1998. Conservation targets in South American Temperate forests. Science 282: 1271-1272.

Anderson CB, GE Likens, R Rozzi, JR Gutiérrez, JJ Armesto, A Poole. 2008. Integrating science and society through longterm socio-ecological research. Environmental Ethics 30: 295-312.

Anderson CB, R Rozzi, JJ Armesto, JR Gutiérrez. 2010. Introduction building a Chilean network for long-term socioecological research: advances and perspectives and relevance. Revista Chilena de Historia Natural 83: 1-11.

Anderson CB, JL Celis-Diez, BJ Bond, G Martínez Pastur, C Little, JJ Armesto, C Ghersa, A Austin, T Schlichter, A Lara, M Carmona, EJ Chaneton, JR Gutiérrez, R Rozzi, K Van- derbilt, G Oyarce, RJ Fernández. 2012. Progress in creating a joint research agenda that allows networked longterm socio-ecological research in southern South America: Addressing crucial technological and human capacity gaps limiting its application in Chile and Argentina. Austral Ecology 37: 529-536.

Bannister JR, OJ Vidal, E Teneb, V Sandoval. 2012. Latitudinal patterns and regionalization of plant diversity along a 4270-km gradient in continental Chile. Austral Ecology 37: 500-509.

Gutiérrez AG, A Huth. 2012. Successional stages of primary temperate rainforests of Chiloé Island, Chile. Perspectives in Plant Ecology, Evolution and Systematics 14: 243-256.

Haberl H, V Winiwarter, K Andersson, RU Ayres, C Boone, A Castillo, G Cunfer, M Fischer-Kowalski, WR Freudenburg, E Furman, R Kaufmann, F Krausmann, E Langthaler, H Lotze Campen, M Mirtl, CL Redman, A Reenberg, A Wardell, B Warr, H Zechmeister. 2006. From LTER to LTSER: conceptualizing the socioeconomic dimension of long-term socioecological research. Ecology and Society 11: 13.

Hedin LO, JJ Armesto, AH Johnson. 1995. Patterns of nutrient loss from unpolluted, old-growth temperate forests: Evaluation of biogechemical theory. Ecology 76: 493-509.

Knight AT, Cowling RM, Campbell BC. 2006. An operational model for implementing conservation action. Conservation Biology 20: 408-419.

Sunderland T, J Sunderland-Groves, P Shanley, B Campbell. 2009. Bridging the Gap: how can information access and exchange between conservation biologists and field practitioners be improved for better conservation outcomes? Biotropica 41: 549-554. 
